\title{
Orthologous relationship of obscurin and Unc-89: phylogeny of a novel family of tandem myosin light chain kinases
}

Received: 16 January 2004 / Accepted: 26 April 2004 / Published online: 5 June 2004

C) Springer-Verlag 2004

\begin{abstract}
Myosin light chain kinases (MLCK) are a family of signaling proteins that are required for cytoskeletal remodeling in myocytes. Recently, two novel MLCK proteins, SPEG and obscurin-MLCK, were identified with the unique feature of two tandemlyarranged MLCK domains. In this study, the evolutionary origins of this MLCK subfamily were traced to a probable orthologue of obscurin-MLCK in Drosophila melanogaster, Drosophila Unc-89, and the MLCK kinase domains of zebrafish SPEG, zebrafish obscurin-MLCK, and human SPEG were characterized. Phylogenetic analysis of the MLCK domains indicates that the carboxy terminal kinase domains of obscurin-MLCK, SPEG and Unc-89 are more closely related to each other than to the amino terminal kinase domains or to other MLCKs, supporting the assertion that obscurin-MLCK is the vertebrate orthologue of Caenorhabditis elegans Unc-89, a giant multidomain protein that is required for normal myofibril assembly. The apparent lack of an invertebrate orthologue of SPEG and the conserved exon structure of the kinase domains between SPEG and obscurin-MLCK suggests that SPEG arose from obscurin-MLCK by a gene duplication event. The length of the primary amino acid sequence between the immunoglobulin (Ig) domains associated with the MLCK motifs is conserved in obscurin-MLCK, SPEG and C. elegans Unc-89, suggesting that these putative protein interaction domains may target the kinases to highly conserved intracellular sites. The conserved arrangement of the tandem MLCK domains and their relatively restricted expression in striated muscle indicates that
\end{abstract}

Edited by D. Tautz

S. B. Sutter · M. O. Raeker · A. B. Borisov · M. W. Russell $(\bowtie)$

Department of Pediatrics and Communicable Diseases,

Division of Pediatric Cardiology, University of Michigan,

1242 Women's Hospital, 1500 E. Medical Center Dr.,

Ann Arbor, MI 48109, USA

e-mail: mruss@umich.edu

Tel.: +1-734-7645177

Fax: +1-734-9369470 further characterization of this novel MLCK subfamily may yield important insights into cardiac and skeletal muscle physiology.

Keywords Obscurin - Muscle development . Myofibrillogenesis $\cdot$ Sarcomere $\cdot$ SPEG

\section{Introduction}

Assembly of the sarcomere, the smallest contractile unit of striated muscle cells, occurs during embryonic differentiation of heart and skeletal muscle, and during continuous myofibril turnover throughout the lifetime of the organism. Increased organization of contractile proteins into sarcomeres occurs in response to physiologic stimuli such as exercise and somatic growth, and pathologic stimuli such as hypertension, myocardial infarction and heart failure. These stimuli elicit the hypertrophic response through reactivation of fetal gene expression, increased protein synthesis and activation of specific protein kinase cascades. The activation of kinase cascades has attracted particular interest due to their differential activation by physiologic and pathologic stimuli (Sugden 2001), and their potential use as targets of therapeutic intervention. However, how activation of these kinase cascades leads to specific cytoskeletal alterations noted during hypertrophic remodeling and myofibrillogenesis is less well understood.

Potential downstream effectors of these cascades that may be directly responsible for producing the cytoskeletal alterations that allow for the assembly and integration of new myofibrils include the myosin light chain kinase (MLCK) family of signaling molecules. MLCK family members play important roles in the structure and regulation of the actin-based cytoskeleton. In cultured rat ventricular myocytes and in adult rat heart in vivo, hypertrophic agonists phenylephrine and angiotensin II cause rapid organization of the sarcomere through calci$\mathrm{um} /$ calmodulin- $(\mathrm{Ca} / \mathrm{CaM})$ dependent phosphorylation of myosin light chain $2 \mathrm{v}$ by skeletal muscle MLCK (Aoki et al. 2000). In smooth and skeletal muscle, MLCK catalyzes 
the $\mathrm{Ca} / \mathrm{CaM}$-regulated phosphorylation of the regulatory light chains (RLC) of myosin, resulting in the onset of or augmentation of cellular contraction (Kamm and Stull 2001; Soderling and Stull 2001). Similarly, in non-muscle cells, RLC phosphorylation can lead to an array of cytoskeletal alterations, including cell contraction, cell motility, and the formation of focal adhesions and stress fibers.

In addition to the skeletal muscle and smooth muscle MLCK genes, there are other structural and signaling molecules that possess MLCK-like domains (Table 1). These include the multifunctional kinases such as Trio, and the giant MLCK such as titin, projectin, and stretchin (Kamm and Stull 2001). The hallmark protein motifs of the MLCK family are the immunoglobulin (Ig), Fibronectin3 (Fn3), and serine/threonine kinase (MLCK) domains. The Ig and Fn3 domains interact with other proteins, anchoring the kinase to specific intracellular sites and enabling interactions with specific target molecules. They can also provide a physical separation between functional domains of the protein, allowing spatially distinct signaling interactions within a large protein assembly. Recently, a novel MLCK family member, striated muscle preferentially expressed gene (SPEG), was identified, and found to contain two tandem MLCK motifs (Hsieh et al. 2000). The identification of a second tandem MLCK protein, obscurin-MLCK (Russell et al. 2002), signaled that the tandem MLCK motif was not unique to SPEG, and helped define a new MLCK subfamily. In adult vertebrates, both obscurin-MLCK and SPEG are predominantly expressed in striated cardiac and skeletal muscle and are the only known vertebrate members of the MLCK family to have dual kinase motifs, suggesting that the tandem MLCK arrangement may be of specific importance to striated muscle physiology.

In this paper, we trace the origins of this new subfamily of MLCKs, and examine the phylogenetic relationships between Unc-89, obscurin-MLCK and SPEG. Comparison of the amino acid sequence of the MLCK domains support the assertion that obscurin-MLCK is the vertebrate orthologue of Unc-89. Furthermore, similarities in the exon structure of their serine-threonine kinase domains suggest that SPEG arose from the kinase domains of obscurin-MLCK by a gene duplication event prior to the evolution of higher vertebrates. The conserved arrangement of the tandem kinase domains and the adjoining Ig and Fn3 domains suggests that the SPEG and obscurinMLCK kinases have highly conserved, and potentially overlapping, phosphorylation targets. Identification of the regulatory targets of this new subfamily of MLCK may be vital to our understanding of cytoskeletal remodeling in vertebrate striated muscle, particularly the heart, in response to physiologic or pathologic stress.
Table 1 Myosin light chain kinase (MLCK) family members

Accession number

\begin{tabular}{|c|c|c|}
\hline & & Accession number \\
\hline \multicolumn{3}{|l|}{ MLCKs } \\
\hline Vertebrate & $\begin{array}{l}\text { Human skeletal MLCK } \\
\text { Rat skeletal MLCK } \\
\text { Human sm/nm MLCK }\end{array}$ & $\begin{array}{l}\text { AJ272502 } \\
\text { NP_476557 } \\
\text { Q15476 }\end{array}$ \\
\hline \multicolumn{3}{|c|}{ Tandem kinase family members } \\
\hline $\begin{array}{l}\text { Inverte- } \\
\text { brate }\end{array}$ & $\begin{array}{l}\text { Drosophila Unc- } 89 \\
\text { Caenorhabditis elegans Unc- } \\
89\end{array}$ & $\begin{array}{l}\text { Pending } \\
\text { WP:CE30426 } \\
\text { (Wormbase) }\end{array}$ \\
\hline Vertebrate & $\begin{array}{l}\text { Mouse SPEG } \\
\text { Human SPEG } \\
\text { Zebrafish SPEG } \\
\text { Human obscurin-MLCK } \\
\text { Mouse obscurin-MLCK } \\
\text { Zebrafish obscurin-MLCK }\end{array}$ & $\begin{array}{l}\text { AF215896 } \\
\text { AY603755 } \\
\text { AY578914 } \\
\text { NP443075 } \\
\text { AY603754 } \\
\text { AY603753 }\end{array}$ \\
\hline \multicolumn{3}{|c|}{$\begin{array}{l}\text { Extended family members } \\
\text { Multifunctional proteins }\end{array}$} \\
\hline $\begin{array}{l}\text { Inverte- } \\
\text { brate }\end{array}$ & Drosophila Trio & BAA96093 \\
\hline $\begin{array}{l}\text { Vertebrate } \\
\text { Giant MLC }\end{array}$ & $\begin{array}{l}\text { Human Trio } \\
\text { KS }\end{array}$ & AAC 34245 \\
\hline Inverte- & Drosophila titin & AAF44704 \\
\hline brate & $\begin{array}{l}\text { C. elegans twitchin } \\
\text { Drosophila stretchin-MLCK } \\
\text { Drosophila projectin }\end{array}$ & $\begin{array}{l}\text { CAA33463 } \\
\text { NM_166128 } \\
\text { AAC } 27550\end{array}$ \\
\hline Vertebrate & $\begin{array}{l}\text { Human titin } \\
\text { Mouse titin }\end{array}$ & $\begin{array}{l}\text { NM_003319 } \\
\text { AAH } 25840\end{array}$ \\
\hline
\end{tabular}

\section{Materials and methods}

Computational database searches

Databases at the National Center for Biotechnology Information (http://www.ncbi.nlm.nih.gov/BLAST/Genome/FlyBlast.html, http://www.ncbi.nlm.nih.gov/blast/tracemb.html, http://www.ncbi nlm.nih.gov/genome/seq/DrBlast.html, and http://www.ncbi.nlm. nih.gov/BLAST/) at the National Institutes of Health, The Wellcome Trust Sanger Institute (http://www.ensembl.org/Danio_rerio/blastview), and WormBase Release WS105 (http://www.wormbase.org/ $\mathrm{db} /$ searches/blat) were searched for MLCK-like sequences. The human obscurin-MLCK carboxy terminal kinase domain (aa 1,314 $1,566)$ was used as the protein query sequence in gapped BLASTP and TBLASTN searches of non-redundant nucleotide (nt), protein, transcript, and genomic DNA databases using default search parameters.

\section{Assembly of putative coding sequences}

The cloning of the human and mouse obscurin-MLCK tandem MLCK isoforms and murine SPEG have been previously reported (Hsieh et al. 2000; Young et al. 2001; Russell et al. 2002). Database search identified previously unpublished isoforms of $C$. elegans Unc-89 that included a tandem MLCK domain either as part of a larger Unc-89 transcript (C09D1.1b) or expressed independently (C09D1.1c) (Small et al. 2002). In Drosophila, a putative gene fragment (CG30171, GenBank accession number NM138022) with amino acid sequence similarity to obscurin-MLCK was noted to be immediately $3^{\prime}$ of a putative gene fragment (CG30175, GenBank accession number NM16646) with similarity to the obscurin 
RhoGEF domain. PCR primers were designed to amplify a CG30175-CG30171 transcript from an adult Drosophila cDNA library (Table 2). The resulting composite cDNA sequence was assembled from the CG30175, the CG30175-30171 amplicon, and CG30171 sequences (GenBank accession number pending). Other closely related Drosophila genes identified by the sequence homology search include projectin $\left(E\right.$-score $\left.=9 \mathrm{e}^{-19}\right)$, ribosomal protein S6 kinase $\left(E\right.$-score $\left.=3 \mathrm{e}^{-18}\right)$, and a KIN family protein kinase $\left(E\right.$-score $\left.=2 \mathrm{e}^{-21}\right)$.

Putative exons from the zebrafish obscurin-MLCK isoform and from the zebrafish SPEG gene were identified based on TBLASTN search of the zebrafish genomic database (http://www.ensembl.org/ Danio rerio/blastview) using the complete human obscurin-MLCK tandem kinase isoform and murine SPEG amino acid sequence as probes. PCR primers (Table 2) were designed to amplify the coding sequences of both genes from 3.5 day post-fertilization (obscurin) and adult (SPEG) zebrafish mRNA. The sequences from the overlapping amplicons were assembled to create a composite putative coding sequence. The composite cDNA sequences for both zebrafish SPEG and the MLCK region of zebrafish obscurinMLCK were deposited in GenBank (accession numbers AY578914 and AY603753). As with murine SPEG, zebrafish SPEG was predicted to encode for a protein with six Ig, two Fn3, and two MLCK domains. Similarly, like its human orthologue, zebrafish obscurin-MLCK would be predicted to encode for multiple tandemly-arranged Ig domains, a RhoGEF domain, an Fn3 domain, and two MLCK domains.

The human SPEG gene was constructed from transcripts KIAA1297, APEG and Hs2 5560336461 (http://www.ensembl.org). The KIAA1297 transcript (Gen̈Bank accession number AB037718), which encodes for both MLCK, both Fn3 and four of the Ig domains, was used to predict the amino acid sequence of the human SPEG MLCK region. The constructed putative amino acid sequence was confirmed by comparison to the murine SPEG sequence (GenBank accession number AF215896).

Identification and phylogenetic analysis of MLCK domains

Functional domains were identified by CD-search (A Conserved Domain Database and Search Service, v1.62; http://www.ncbi.nlm. nih.gov/Structure/cdd/cdd.shtml), which uses sequence comparison to the Smart and Pfam collections of structural and functional domains. The tandem MLCK domains were aligned using CLUSTALW (http://www.ebi.ac.uk/clustalw/). The alignment was graphically displayed using MegAlign (DNAStar). The phylogenetic tree was obtained using the neighbor-joining method (MEGA2). Two-thousand bootstrap replications were performed. Bootstrap support values for each branch point were calculated.

\section{Results and discussion}

Conservation of the tandem MLCK domains across species

To compare the degree of sequence conservation between MLCK family members, the protein sequences of the kinase domains were aligned (Fig. 1). The kinase domains of MLCK family members ranged between 235 and 285 aa in length. Across species, we found that the amino terminal kinase of zebrafish SPEG was $61 \%$ identical to the corresponding domains in mouse and human. The carboxy terminal kinase was $37 \%$ and $53 \%$ identical to the carboxy terminal kinase of mouse and human, respectively. Zebrafish obscurin-MLCK kinase domains are 42 $55 \%$ identical to the corresponding domains in the mouse and human orthologues.

We produced a phylogenetic tree using a nearestneighbor algorithm (Fig. 2) to study the evolutionary relationship between the tandem MLCK family members. The tree, rooted with DAP1K, a serine-threonine kinase related to the MLCK family, demonstrated that the amino terminal kinase domains of SPEG and obscurin-MLCK are most closely related to their own vertebrate orthologues and next most closely related to each other. The carboxy terminal kinase domains are more closely related to the corresponding domains of the invertebrate Unc-89 genes than they are to the amino terminal kinase domain or to other MLCKs, suggesting that the MLCK tandem kinase family of genes may have evolved from Unc-89. Furthermore, the more distant relationship between the amino and carboxy terminal kinase domains suggests that if one of the tandem kinases arose from the other by a gene duplication event, it did so early in invertebrate evolution, or there was significant evolutionary pressure to stimulate their divergence.

Conservation of the arrangement of functional domains in the tandem myosin light chain kinase subfamily

For the Unc-89, SPEG, and obscurin-MLCK genes, the arrangement of their functional domains in the MLCK region is highly similar (Fig. 3a). An Ig domain is present

Table 2 Primers used to amplify zebrafish SPEG and obscurin-MLCK

\begin{tabular}{llll}
\hline PCR primers & Forward & Reverse & Product size \\
\hline ZFSPEG3 & AGCTCCCCCTGTATTTCTCC & CAGCCTTCCCCACCTTGTTG & 3 \\
ZFSPEG2 & TGGTACCACAATGGCAAGAA & ATCCAGCAGTTCGGATATGG & 2.03 \\
ZFSPEG1A & TGATGGGCTCATTCACAAGA & CAGAAGGAGAGACGGGAGTG & 1.1 \\
ZFSPEG1B & CTCCCGTCTCTCCTTCTGTG & GTCTGTCGACGCAGCTTTT & 0.928 \\
ZFObtoM & CACCATCCTGAGTCAAAAAGAGT & TAGGGTGGCCAGTAATAATAGC & 1.732 \\
ZFObSkII & CATGACGTGGAGAGGCAGGAGTC & GATGGCCTCTTTTCTGGGTCTG & 1.160 \\
ZFObSkIItoSkI & GTGGAGGCACAGGACTTTCTSCATAG & GACCTCCAGGTACAGTGATCTTTGAC & 2.3 \\
ZFObSkI & CATGGCAATACCTTATGAAGACTC & GTCCTAGTTACACGTCCCACATA & 2.340 \\
\hline
\end{tabular}


Fig. 1 Sequence alignment of serine-threonine kinase domains. The amino (NH2) and carboxy terminal $(\mathrm{COOH})$ serine-threonine kinase domains of ZFSPEG (ZFSPEG NH2 and ZFSPEG COOH, respectively) were aligned with MLCK family members using MegAlign (DNAStar) and ClustalW. Family members include Caenorhabditis elegans Unc-89 (CE Unc-89) isoform b, deathassociated protein kinase 1 (DAP1 K), trio (Trio), smooth muscle/non-muscle MLCK (sm nm MLCK), human SPEG (hum $\overline{S P} \bar{E} G$ ), mouse obscurinMLCK (MusObsMLCK), human obscurin-MLCK ( $h u$ mObsMLCK), Drosophila Unc89 (Dro_Unc-89), and mouse SPEG (musSPEG). Refer to Table 1 for GenBank accession numbers. Shaded boxes highlight residues that are identical to $\mathrm{sm} / \mathrm{nm}$ MLCK. An asterisk denotes residues identical in all kinases. The 15 amino acids conserved in most serine-threonine kinases are denoted with an arrowhead
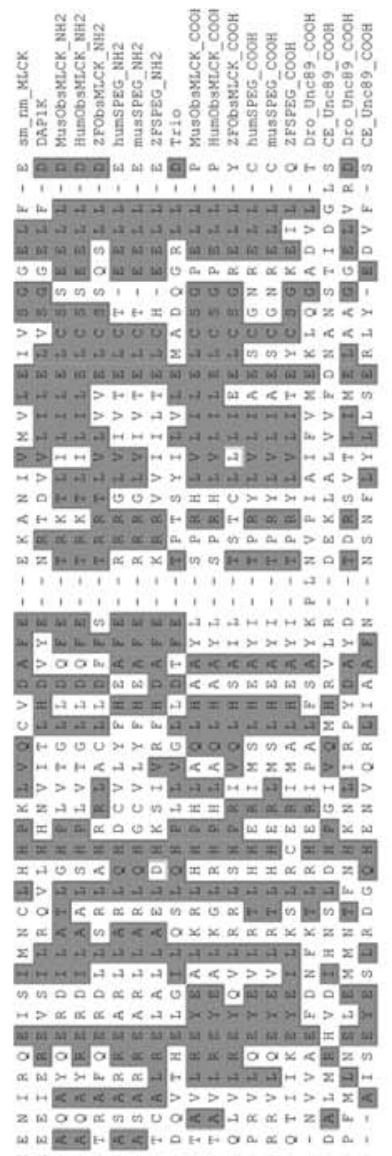

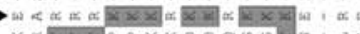

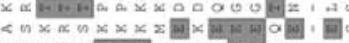

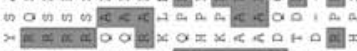
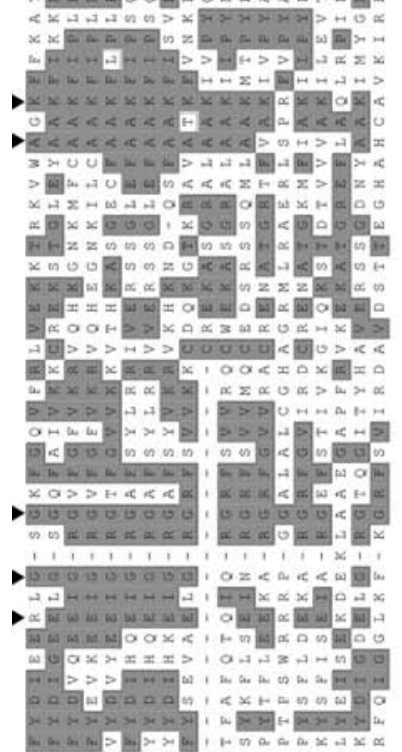

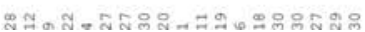
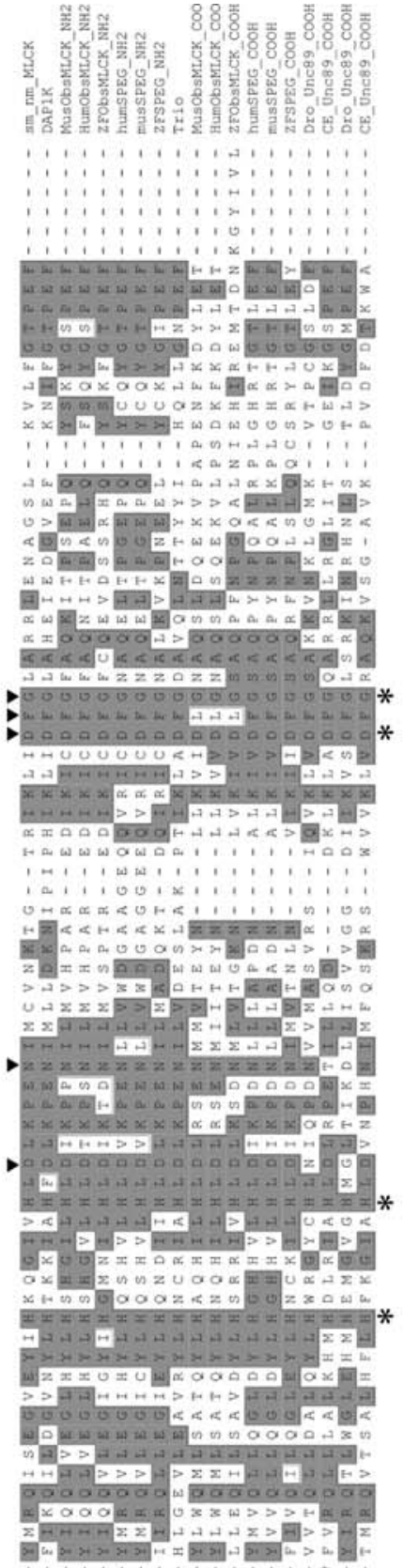

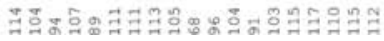

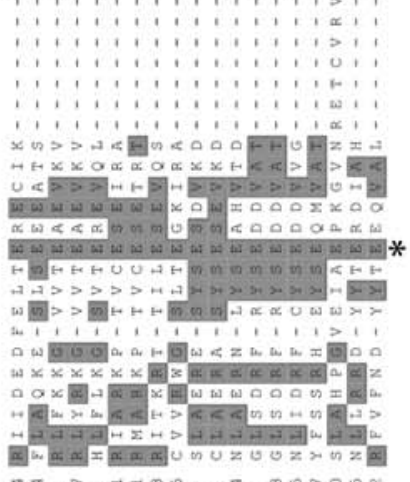

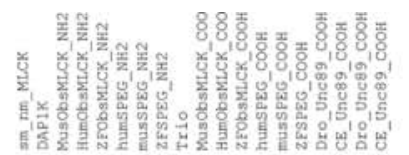

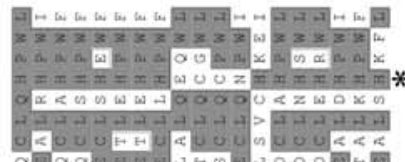

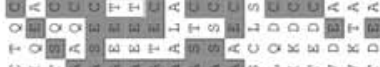
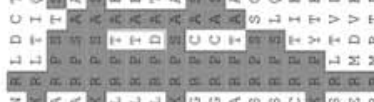

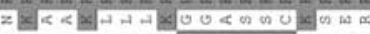

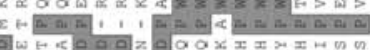

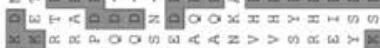

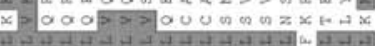
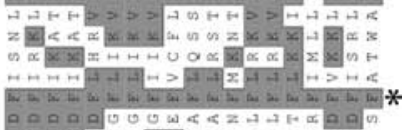

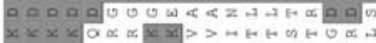

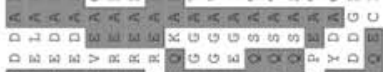

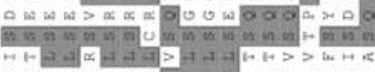

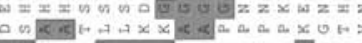

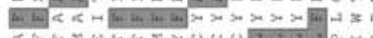

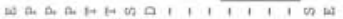

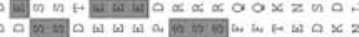

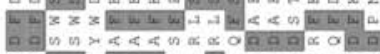

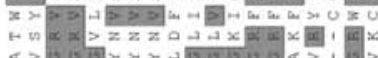

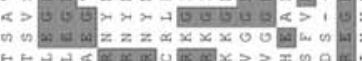

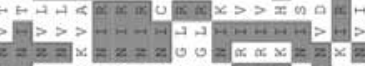

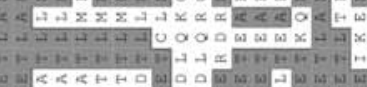

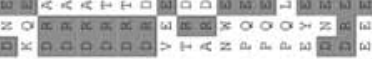

国

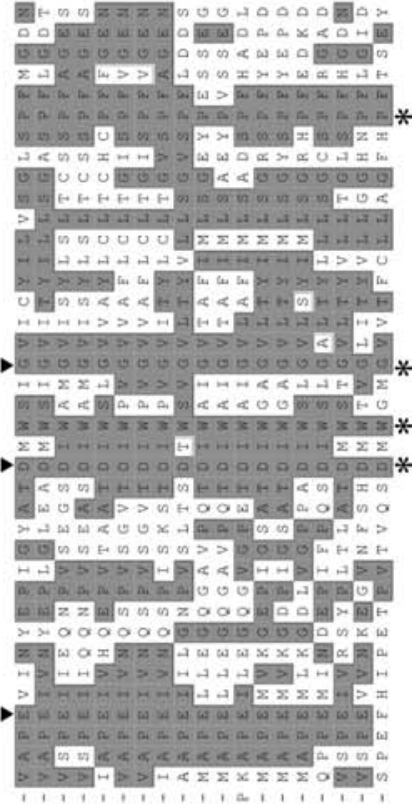

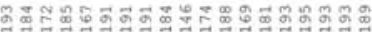




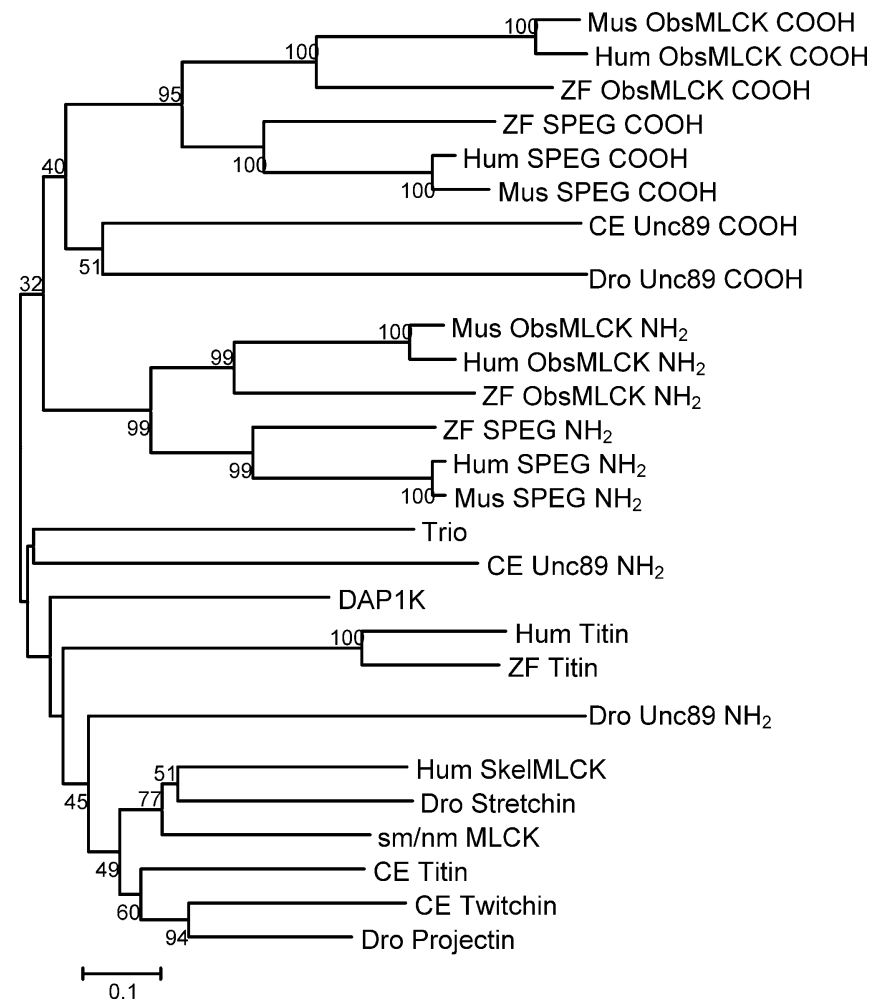

Fig. 2 Rooted phylogenetic tree showing the evolutionary relationships between the kinase domains of MLCK family members. Amino acids were aligned using MEGA2. Bootstrap values greater than $40 \%$ are shown on the branches, based on 2,000 bootstrap replications. See Fig. 1 for details. Additional family members are human skeletal muscle MLCK (HumSkelMLCK), Caenorhabditis elegans titin (CE_Titin), C. elegans twitchin (CE_Twitchin), Drosophila projectin (Dro Projectin), human and zebrafish titin (Hum_titin and ZF_titin, respectively)

immediately upstream of the amino terminal kinase and, sequentially, there is an Ig, Fn3, and tandem MLCK motif at the carboxy terminus of each protein. With respect to the primary amino acid sequence, the distance between the tandem kinase domains is conserved between the $C$. elegans Unc-89 gene and the known vertebrate obscurinMLCK genes, suggesting that the distance between the kinases is essential for their proper function. The amino acid sequence distance between the tandem MLCK domains in Drosophila Unc-89 gene is comparatively less. However, how this difference in the primary sequence affects the three dimensional structure and true distance between the kinase domains is not known. The primary amino acid sequence distance between the kinase domains is somewhat longer for SPEG but is consistent across species.

The exon structures of the MLCK-encoding regions of the SPEG and obscurin-MLCK genes are remarkably similar with the only significant differences involving an exon immediately $5^{\prime}$ of the carboxy terminal MLCK domain (Fig. 3b). This exon is approximately $80 \mathrm{nt}$ in all obscurin-MLCK genes and approximately $600 \mathrm{nt}$ in all known SPEG genes. The increased length of the resulting primary amino acid sequence would be predicted to increase the physical separation between the carboxy terminal kinase and the accompanying Ig and Fn3 domains. This increased distance appears to be one of the fundamental differences between the SPEG and obscurin-MLCK. Interestingly, although the distances between the kinase domains differ between obscurinMLCK and SPEG, the distances between the Ig domains upstream of each amino terminal kinase domain are very similar (data not shown). The similar distances between the potential protein-interaction domains, Ig and Fn3, suggests that SPEG and obscurin-MLCK may interact with similar scaffolding complexes within the sarcomere; however, their targets of phosphorylation may differ due to the altered relationship between the carboxy terminal kinase domain and its associated Ig-Fn3 domains.

Relationship of the vertebrate obscurin-MLCK to the invertebrate Unc-89

The identification of rare tandem MLCK motifs in both Unc-89 and obscurin-MLCK provides the most definitive evidence to date that obscurin-MLCK is the vertebrate orthologue of Unc-89. That the Unc-89 amino and carboxy terminal MLCK domains are most closely related to the corresponding obscurin-MLCK domains, and that both genes contain multiple tandem Ig, a RhoGEF and tandem MLCK domains supports this assertion. To date, no other invertebrate or vertebrate proteins have been identified with this unique constellation of signaling and adhesion domains.

One interesting difference between Unc- 89 and obscurin is the location of the RhoGEF domain. In Unc-89, the RhoGEF domain is located at the N-terminus of the protein, while in obscurin-MLCK, the domain is located carboxy terminal to the tandem Ig domains and amino terminal to the MLCK domains. This suggests that a major genomic rearrangement of the Unc-89 gene occurred prior to, or in the process of, giving rise to the obscurin-MLCK gene. How this rearrangement affects the spatial separation of the MLCK and RhoGEF domains and whether or not this affects the properties of the protein is not currently known.

Establishing an evolutionary relationship between Unc89 and obscurin-MLCK allows the extrapolation of studies on Unc-89 function in invertebrates to model the potential functions of obscurin-MLCK in vertebrates. Unc-89, a $732-\mathrm{kDa}$ polypeptide that localizes to the M-band in mature myocytes was first identified in C. elegans (Benian et al. 1996). In Unc- 89 mutants, the myofibrils were highly disorganized and lacked an M-band in most mutant alleles (Waterston et al. 1980). It was proposed that the long tandem array of Ig domains in Unc-89 might interact with other proteins in the thick filament assembly and help to organize the myofilament through the recruitment and spatial ordering of myofibrillar and cytoskeletal proteins. Furthermore, since interactions between the M-band structures and the cell membrane (and subsequently the extracellular matrix) appear to be necessary for proper 


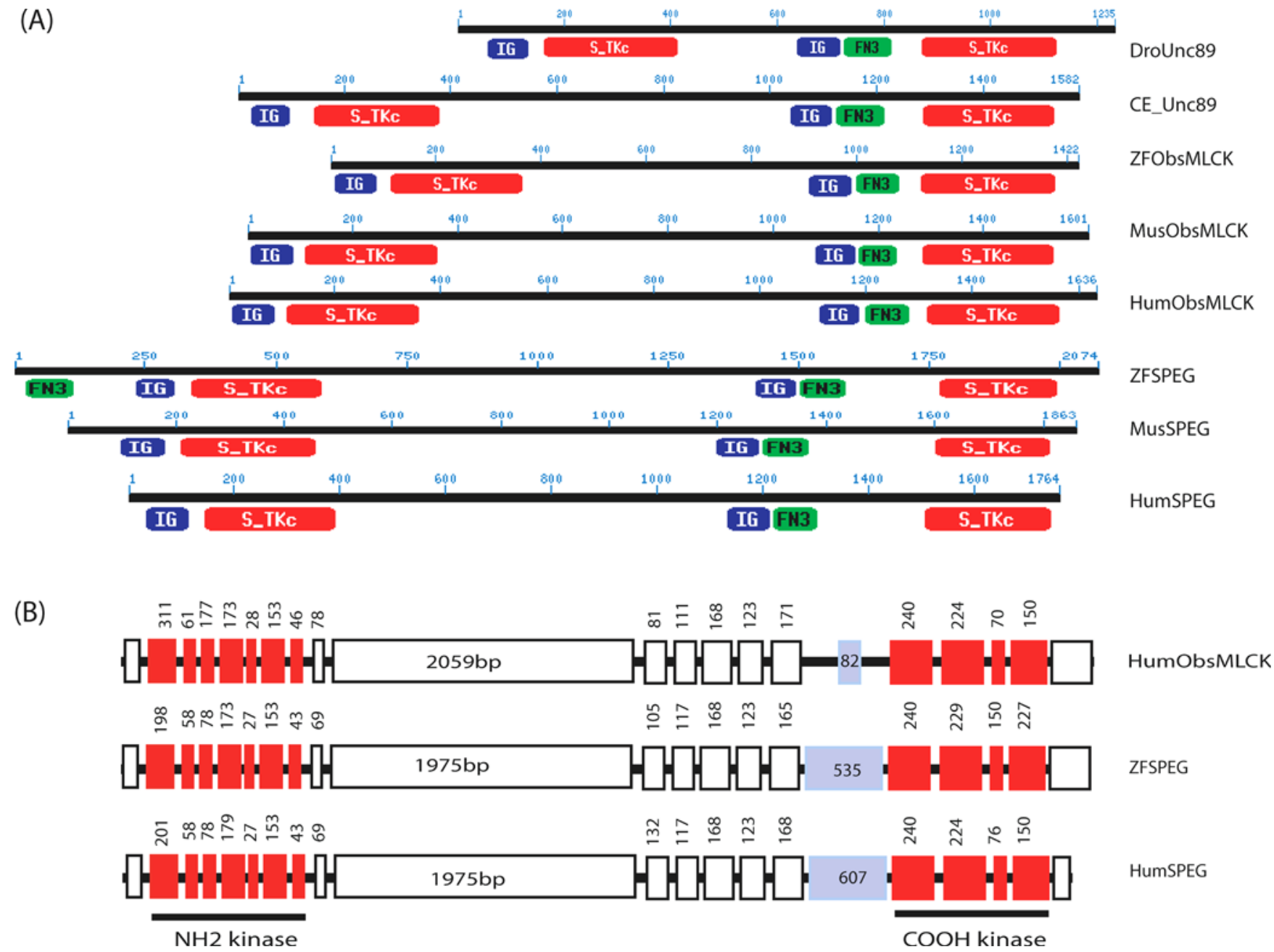

Fig. 3 a Functional domains of kinase domains of ZFSPEG and other MLCK family members: immunoglobulin domains (Ig), serine-threonine kinase domain $(S T K c)$, fibronectin type III $(F n 3)$. b Exon structure of the tandem MLCK domains of the zebrafish SPEG and human obscurin-MLCK and SPEG genes. Exons are shown as boxes. Those exons encoding for the MLCK are denoted in red and the exon that varies in length between SPEG and

myofibril assembly, it was proposed that Unc-89 may be an important mediator in this process.

What has been determined to date about obscurin supports both the assertion that it is the orthologue of Unc89 and that it has a role in myofibril organization. Expressed primarily in the heart (similar to the pharyngeal musculature in C. elegans) and skeletal muscle in the adult organism, obscurin (lacking the tandem kinase domain) localizes to the M-band as the sarcomere matures from the pre-myofibrillar state to the mature striated pattern (Bang et al. 2001; Russell et al. 2002). In remodeling myocytes in culture, it appears to span the distance between adjacent but not yet fused myofibrils, potentially aiding their lateral alignment into an organized register (Borisov et al. 2004). As myofibrils are generated in vitro in response to a hypertrophic signal, obscurin intensely localizes to the new sites of myofibrillogenesis (Borisov et al. 2003). Recently described interactions between obscurin and titin (Bang et al. 2001; Young et al. 2001) and between
obscurin-MLCK is denoted in purple. Size is indicated in base pairs. In this diagram the introns have been removed and the spaces between the exons do not represent intron lengths. Note that the exon arrangement is highly similar in all three genes, indicating conservation of the exon structure both across species and between SPEG and obscurin-MLCK

obscurin and ankyrin (Bagnato et al. 2003; KontrogianniKonstantopoulos et al. 2003) further support the model that obscurin may serve as a vital link between the myofibrillar apparatus and the cell membrane/extracellular matrix, as was proposed for Unc-89.

The relationship between the MLCK domains and the RhoGEF/Ig region in both Unc-89 and obscurin-MLCK remains to be determined. For both obscurin and Unc-89, a large isoform containing only the RhoGEF domain and the Ig region can, and often is, expressed without the MLCK domains (Benian et al. 1996; Russell et al. 2002). Furthermore, the obscurin-MLCK domains appear to be most commonly expressed without the large, RhoGEFcontaining region. To date, there is no report of independent expression of the MLCK domains of Unc-89. Given the uncertainty of how to refer to the alternatively-spliced forms of obscurin, we would like to propose a nomenclature for the different isoforms based on what is known to date (Fig. 4). 
Fig. 4 Schematic representation and proposed nomenclature of the tandem MLCK family and their known isoforms. Represented motifs include the Ig, Fn, CaM-binding, Rho and MLCK domains. The KSP repeat region is unique to Unc-89

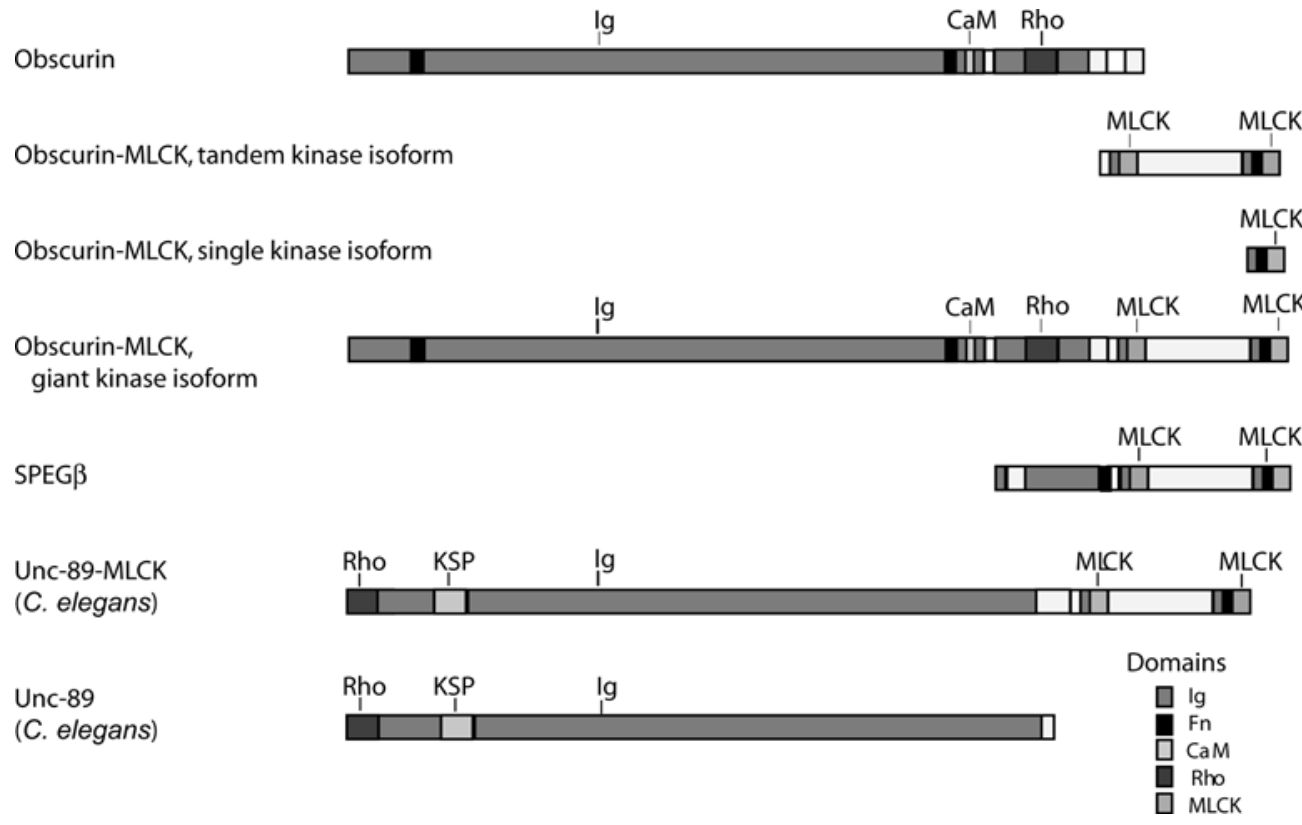

Interestingly, preliminary studies would suggest that the smaller, MLCK-only isoforms of obscurin-MLCK may have a distinctly different cellular localization pattern from the large obscurin. In mature myocytes, the carboxy terminal kinase domain of the obscurin-MLCK isoform localizes predominantly to the Z-band and to the nucleus in mature myofibrils (data not shown). The different primary sites of action of the two major obscurin isoforms may have necessitated independent expression of the MLCK domains.

\section{Relationship of obscurin-MLCK and SPEG}

The Z-band localization pattern of obscurin-MLCK isoforms is similar to what has been noted for SPEG. Like obscurin-MLCK, SPEG is predominantly expressed in striated muscle, although some non-kinase-containing isoforms (APEG and BPEG) are preferentially expressed in the aorta and brain, respectively (Hsieh et al. 2000). In myocytes, SPEG is not detected in myoblasts but is induced during myofibrillogenesis, localizing to the Zbands in mature myofibrils. As such, it appears to be at least a marker of, and perhaps required for, the differentiation process. The reason for SPEG's evolution from obscurin-MLCK can only be speculated, given their potential co-localization in mature myocytes. Given the potentially altered spatial relationship between the carboxy terminal kinase domain and the accompanying $\mathrm{Ig} / \mathrm{Fn} 3$ region, it is likely that there are unique targets that can be phosphorylated by only one or the other. Also of interest is the observation that the carboxy terminal kinase of obscurin-MLCK, but not SPEG, can be expressed independent of the amino terminal kinase in a single kinase isoform. The ratio of expression of the obscurin-MLCK single kinase isoform to the tandem kinase isoform is far greater in the mouse heart than the human heart and may be secondary to the important physiologic differences between the two.

\section{Conclusion}

Studies in invertebrates on Unc-89 and in vertebrates on obscurin-MLCK and SPEG indicate that the tandem MLCK family of signaling proteins may be vitally important to myofibrillogenesis and myocyte physiology. Given the capacity for MLCKs to elicit cytoskeletal alterations, it is likely that this family of signaling proteins is involved during the myofibrillar remodeling that accompanies myofibrillogenesis, the response to hypertrophic stimuli, and /or the adaptation to stress. Since the MLCK domains and their expression as a tandem unit is conserved across species, it is presumed that both are functional kinases. It is less clear whether they are concurrently, reciprocally, or independently activated and functional. Additional studies will be required to determine the functions and phosphorylation targets of this family of tandem MLCKs, and to determine the exact role of these proteins during myofibrillogenesis and myofibril remodeling.

Acknowledgements The authors would like to acknowledge Xiaoxia Wang for excellent technical assistance with the phylogenetic tree. This study was supported by funding from NIH 5R03 HD39693-02 to M.W.R.

\section{References}

Aoki H, Sadoshima J, Izumo S (2000) Myosin light chain kinase mediates sarcomere organization during cardiac hypertrophy in vitro. Nat Med 6(2):183-188 
Bagnato P, Barone V, Giacomello E, Rossi D, Sorrentino V (2003) Binding of an ankyrin-1 isoform to obscurin suggests a molecular link between the sarcoplasmic reticulum and myofibrils in striated muscles. J Cell Biol 160(2):245-253

Bang ML, Centner T, Fornoff F, Geach AJ, Gotthardt M, McNabb M, Witt CC, Labeit D, Gregorio CC, Granzier H, Labeit S (2001) The complete gene sequence of titin, expression of an unusual approximately $700-\mathrm{kDa}$ titin isoform, and its interaction with obscurin identify a novel Z-line to I-band linking system. Circ Res 89(11):1065-1072

Benian GM, Tinley TL, Tang X, Borodovsky M (1996) The Caenorhabditis elegans gene unc-89, required for muscle Mline assembly, encodes a giant modular protein composed of Ig and signal transduction domains. J Cell Biol 132(5):835-848

Borisov AB, Raeker MO, Kontrogianni-Konstantopoulos A, Yang K, Kurnit DM, Bloch RJ, Russell MW (2003) Rapid response of cardiac obscurin gene cluster to aortic stenosis: differential activation of Rho-GEF and MLCK and involvement in hypertrophic growth. Biochem Biophys Res Commun 310 (3):910-918

Borisov AB, Kontrogianni-Konstantopoulos A, Bloch RJ, Russell MW (2004) Dynamics of obscurin localization during myofibrillogenesis and remodeling in cardiac myocytes: obscurin as an integrator of sarcomere structure. J Histochem Cytochem (in press)

Hsieh CM, Fukumoto S, Layne MD, Maemura K, Charles H, Patel A, Perrella MA, Lee ME (2000) Striated muscle preferentially expressed genes alpha and beta are two serine/threonine protein kinases derived from the same gene as the aortic preferentially expressed gene-1. J Biol Chem 275(47):36966-36973
Kamm KE, Stull JT (2001) Dedicated myosin light chain kinases with diverse cellular functions. J Biol Chem 276(7):4527-4530

Kontrogianni-Konstantopoulos A, Jones EM, van Rossum DB, Bloch RJ (2003) Obscurin is a ligand for small ankyrin 1 in skeletal muscle. Mol Biol Cell 14(3):1138-1148

Russell MW, Raeker MO, Korytkowski KA, Sonneman KJ (2002) Identification, tissue expression and chromosomal localization of human obscurin-MLCK, a member of the titin and Dbl families of myosin light chain kinases. Gene 282(1-2):237-246

Small TM, Flaherty, Denise B, Mercer, Kristina B, Williams, Teresa M, Benian, Guy M, and Borodovsky, Mark (2002) A new isoform of UNC-89 with two MLCK-like protein kinase domains (abstract). In: East coast worm meeting, 14-16 June, Durham, N.H.

Soderling TR, Stull JT (2001) Structure and regulation of calcium/ calmodulin-dependent protein kinases. Chem Rev 101 (8):2341-2352

Sugden PH (2001) Mechanotransduction in cardiomyocyte hypertrophy. Circulation 103(10): 1375-1377

Waterston RH, Thomson JN, Brenner S (1980) Mutants with altered muscle structure of Caenorhabditis elegans. Dev Biol 77 (2):271-302

Young P, Ehler E, Gautel M (2001) Obscurin, a giant sarcomeric Rho guanine nucleotide exchange factor protein involved in sarcomere assembly. J Cell Biol 154(1):123-136 